\section{A Case of Adenomyosis of the Uterus}

Hima Bindu Ponnam \& Y. S. Suryanarayana, India

\section{Introduction}

Uterine adenomyosis is the benign invasion of endometrial glands and stroma into the myometrium. Most cases (90\%) occur in multiparous women, and about $80 \%$ of women with adenomyosis are 40 to 50 years old. The most frequent symptoms are menorrhagia, dysmenorrhoea, and metrorrhagia. Classically the uterus is diffusely enlarged and tender to palpation. The role of adenomyosis in the pathogenesis of menometrorrhagia is perplexing, in part because it frequently occurs in asymptomatic women [1]. Adenomyosis has been found in 54\% of uteri examined at autopsy [2]. In a prospective study of patients undergoing hysterectomy, there was no difference in duration of menstrual bleeding or presence of dysmenorrhoea in the 28 patients found to have adenomyosis and 157 without adenomyosis or endometriosis [3]. On the other hand, adenomyosis is often associated with abnormal uterine bleeding. Adenomyosis was found in hysteroscopic biopsy specimens in $37 \%$ of 90 menorrhagic women. However, if the uterine cavity was normal, the number of patients who had significant adenomyosis increased to $66 \%$ [4]. Adenomyosis was found in 20 of 43 women with an enlarged uterus without evidence of leiomyomata on ultrasound who had a hysterectomy for persistent menorrhagia [5]. Ultimately, the role of adenomyosis in abnormal uterine bleeding may prove to be quantitative, with more extensive disease and greater depth of myometrial invasion more likely to cause abnormal bleeding [1]

Adenomyosis typically affects women of reproductive age. In general, affected women are multiparous, and the condition is seen with higher frequency in woman with a history of surgical uterine procedures (e.g., Caesarean section, dilatation and curettage). It has a reported incidence ranging widely from 5 to $70 \%$ [6]. Diagnosis can be made with the help of transabdominal and transvaginal ultrasound, hysteroscopy and
MRI. Homeopathy offers a better alternative for the treatment of uterine adenomyosis. The below presented case is one such example where homeopathic medicine proved to be safe and effective in the treatment of uterine adenomyosis by not only improving the general health of the individual but also preserving her uterus.

\section{Case Presentation}

A female patient of 27 years of age has come to the OPD complaining of bleeding per vagina continuously for the past fifteen days. The blood was dark red and clotted. The patient was suffering from irregular menstrual cycles with protracted and profuse bleeding for almost a year. The patient has been on allopathic medication for almost the past six months with no relief. As the patient had already two children and the menorrhagia was devastating and not responding to treatment she was advised to undergo a hysterectomy by the consulting gynaecologist. As the patient was reluctant to undergo surgery and had previously benefitted from homeopathy, she opted for homeopathic therapy. She was referred to the OPD of the Extension Clinical Research
Unit, Princess Durru Shehvar Children's \& General Hospital, Hyderabad by the consultant gynaecologist of the said hospital. Her presenting complaints were:

a) Menstrual cycles appeared every fifteen days.

b) Each cycle lasted for about ten days.

c) Severe pain, abdomen and back, felt during the flow with much exhaustion and fainting.

\section{Past history}

She had an attack of chikun-gunya two years back and had taken an allopathic treatment with relief. The pains subsided within a month. Family history was unremarkable. Parents were not alive and mother died one year back. Patient had four siblings - three sisters, one brother - who were all clinically normal.

She was the third child of non-consanguineous parents. She was educated up to tenth class and discontinued as she was married early. She attained menarche at the age of twelve years. Her menstrual cycles were regular every thirty days, bleeding lasting for five days. She was a housewife and had two children - one male and one female, one was a normal delivery and the second one an emergency caesarean. Her first childbirth was five years back and her last childbirth was three years back.

\section{Physical generals}

- Thermal reaction: Hot patient, desire for cold weather and air

- Desire: Salty food and salt

\section{S U M M A R Y}

Uterine adenomyosis is considered benign and even though not a lifethreatening condition, the frequent pain and heavy bleeding associated with it can have a negative impact on a woman's quality of life. This is a condition seen with higher frequency in women with a history of surgical uterine procedures (e.g., Caesarean section, dilatation and curettage). The only definitive cure for adenomyosis is a hysterectomy. This is often the treatment of choice for women with significant symptoms. This surgical intervention can be avoided through homeopathic treatment. A case of uterine adenomyosis in a twenty-seven-year-old female who presented with continuous heavy bleeding showed the efficacy of homeopathic medicines in giving not only symptomatic relief to the patient but also helping in the complete cure of the adenomyosis of the uterus.

KEYWORDS Menorrhagia, Uterine adenomyosis, Trillium pendulum, Natrum muriaticum 


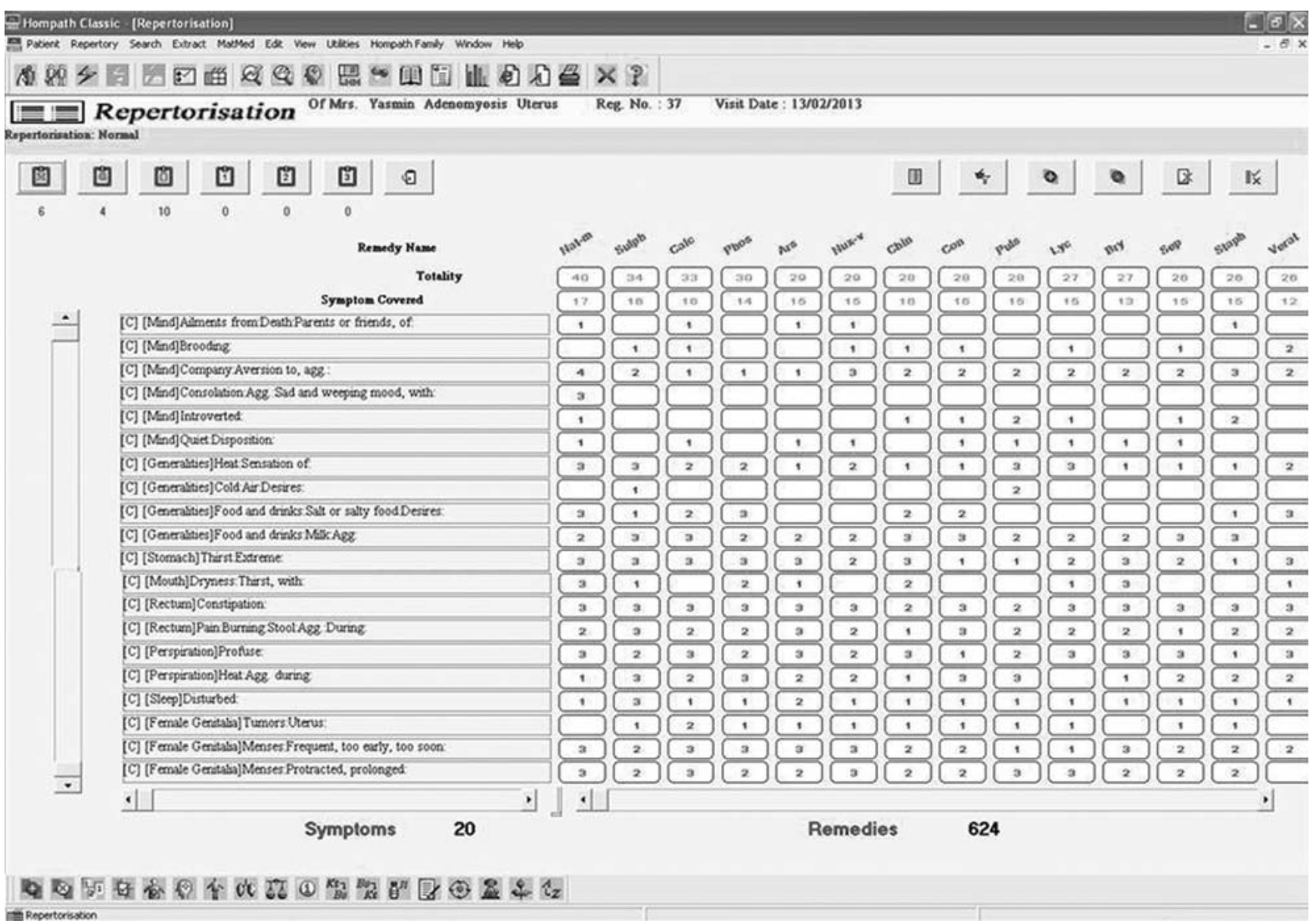

Fig. 1 Repertorisation of the case (Complete Repertory in Hompath Classic).

- Aversion: Nonspecific

- Intolerance: Milk

- Appetite: Diminished

- Thirst: Extreme dryness of mucous membranes and thirst extreme

- Stool: Constipated, alternate days with burning in anus

- Urine: Normal (complained of involuntary urine on sneezing and coughing for the past three months)

- Perspiration: Profuse especially in hot weather, oily

- Sleep: Disturbed due to thoughts of her dead mother

Mental generals

- Desires to be alone

- Sensitive to noises and trifles

- Never weeps before others, but broods when alone secretly

- Consolation by others aggravates her troubles

- Very much stressed after the death of her mother, observed that the present complaint started after the death of her mother
General physical examination

She is medium complexioned, normal built.

Height: 5"6', Weight: $65 \mathrm{~kg}$, BMI (body mass index): 23.

Anaemic - Nil, Jaundice - Nil, Cyanosis Nil, Generalised lymphadenopathy - Nil.

Pulse - 72/min, Temperature - $98.6^{\circ} \mathrm{F}$, Respiratory rate $-12 / \mathrm{min}, \mathrm{BP}-110 / 70 \mathrm{mmHg}$.

Respiratory system: lungs are clear.

Cardiovascular system: S1, S2 normal, no added sounds.

Gastrointestinal system: NAD.

Locomotor system: NAD.

Pelvic examination: Bleeding present.

Routine haemogram: Haemoglobin $8 \mathrm{gms} / \mathrm{dl}$.

USG pelvis shows bulky uterus diagnosed with adenomyosis of uterus (Fig. 2).

\section{Treatment}

Initially Trillium pendulum 30C, one dose every four hours has been prescribed to control bleeding per vagina with pain, severe pain in back, and much exhaustion with fainting as presented by the patient. The case was repertorised on the basis of the Complete Repertory [7] taking into consideration the totality (Fig. 1). Natrum muriaticum was thought to be appropriate for the case, as it was found suitable after repertorisation and in consultation with materia medica.

\section{Follow-Up Criteria}

The case was followed for a period of one year post-menstrually with USG pelvis done at entry and at the end of one year as per the protocol.

1st follow-up: The patient had reported after two days with cessation of bleeding after taking Trillium pendulum 30C. Placebo continued for fifteen days. 
2nd follow-up: At this juncture, the case was analysed, evaluated and repertorised. Natrum muriaticum $30 \mathrm{C}$ in a single dose was prescribed based on the totality, with placebo daily, one dose for a month.

3rd follow-up: After a month with the first dose of Natrum muriaticum 30C, the menses appeared on the 29th day and bleeding lasted for five days. Weakness reduced. Pain in back and abdomen relieved. She was therefore given placebo, daily, one dose for a month.

4th follow-up: The patient reported in the next month post-menstrually. She had menses on the 28th day and bleeding lasted for five days. Pain in back and abdomen reappeared during menses. Natrum muriaticum 30C, one dose was repeated with placebo for one month.

5th follow-up: The patient reported in the next month post-menstrually; had menses on the 30th day and bleeding for five days. Pain in abdomen and back relieved. She was therefore given placebo, daily, one dose for a month.

6th follow-up: The patient reported in the next follow-up post-menstrually; had menses on the 27 th day and bleeding for five days. Pain in abdomen and back reappeared. Natrum muriaticum 200C, one dose with placebo for one month was prescribed.

7th follow-up: The patient reported in the next month post-menstrually; had menses on the 30th day and bleeding for five days. Pain in abdomen and back relieved. She was therefore given placebo, daily, one dose for a month.

8th follow-up: Next month, the menses appeared on the 31st day, bleeding lasted for five days, no complaints. Placebo for one month.

9th follow-up: Next follow up, the patient reported after two months. The last menses came early by two weeks, bleeding was profuse, lasted for five days and spotting lasted for five days. Now Natrum muriaticum 200C, one dose was repeated with placebo, daily, one dose for a month.

10th follow-up: Next follow up, the patient reported after two months. Menses had been all three months every 30 days, bleeding lasted for five days. No other complaints. In this follow-up, USG pelvis has been done for the final assessment of the case.

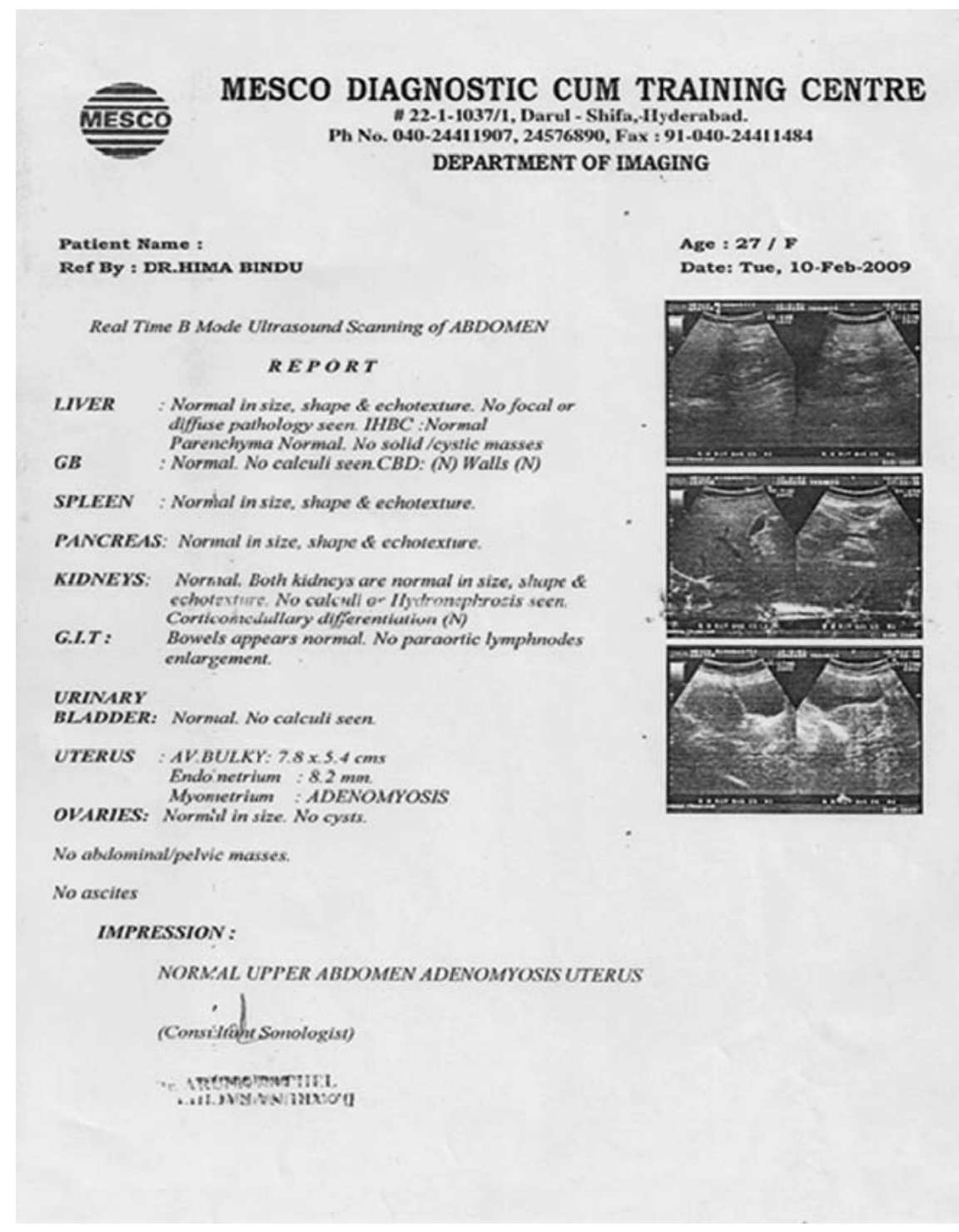

Fig. 2 USG pelvis at entry.

Impression of the report: Uterus is normal, no evidence of any mass lesion inside the uterus, normal pelvic scan (Fig. 3).

\section{Discussion}

The patient presented with adenomyosis uterus in the reproductive age group i.e., 27 years of age and with abnormal uterine bleeding as per the version of McCausland [8]. There is no family history reported in the present case. Abnormal uterine bleeding is the most frequently cited symptom in women with adenomyosis of the uterus as per Stewart's classification system [9] of clinical presentation of uterine adenomyosis and the present case reported with continuous bleeding per vagina as the chief complaint for fifteen days.
When initially the subject approached with the complaint of continuous bleeding per vagina with severe back pain and much exhaustion with fainting, Trillium pendulum was thought to be appropriate as per Boericke [10] and Allen [11]. After giving Trillium pendulum $30 \mathrm{C}$, bleeding per vagina stopped. At this juncture, the case had been taken in detail, analysed, evaluated and repertorised to select a similimum. In the case along with the totality much emphasis was observed on the mental general and causation i.e., ailments after the death of the mother became the guiding symptom. The selection of Natrum muriaticum is thus based on the totality of characteristic symptoms further guided by the keynote that is the prominent mental general. The dose of Natrum muriaticum was administered post-menstrually in this case in accordance with Allen [12]. 


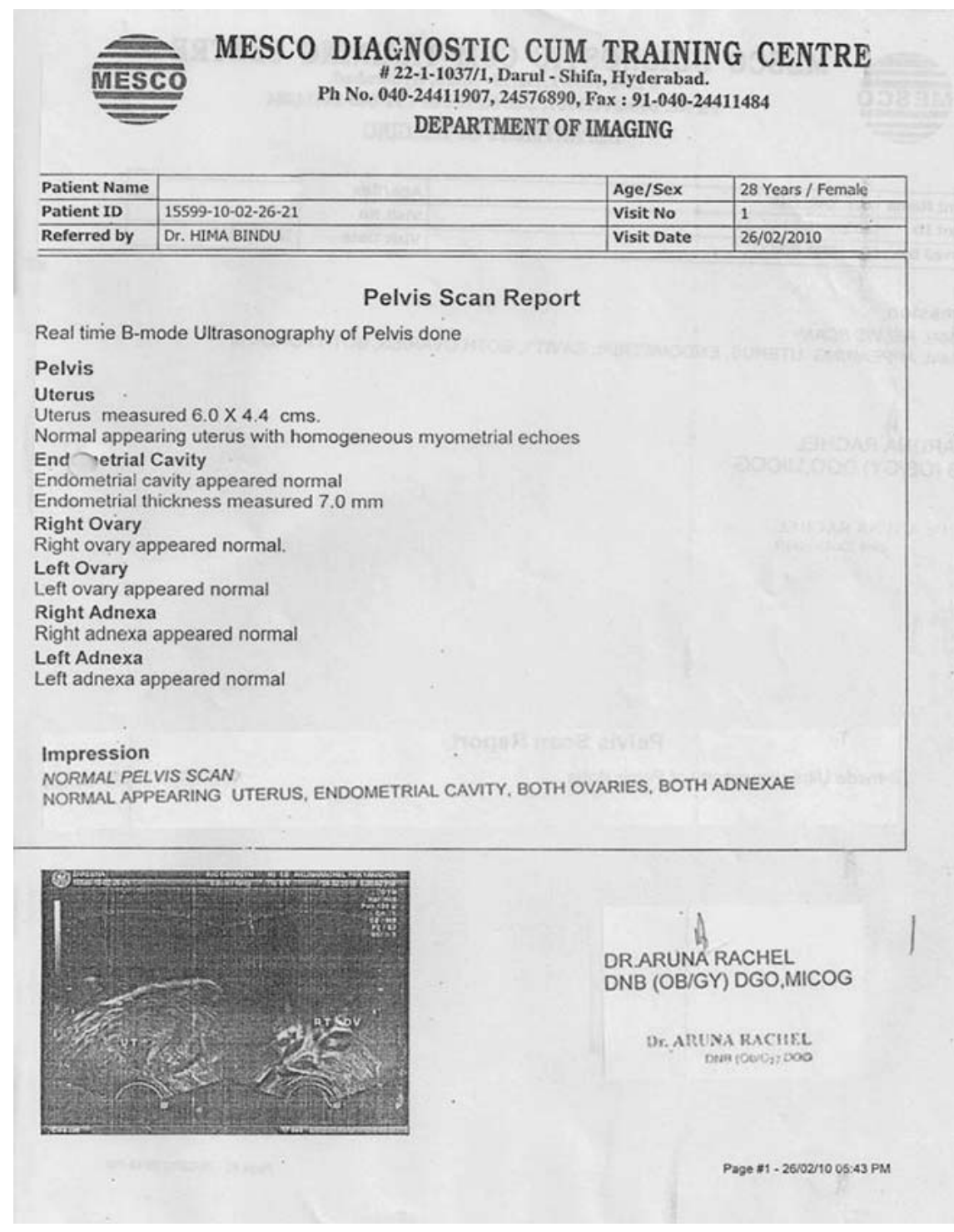

Fig. 3 USG pelvis at end (after 1 year of treatment).

Initially Natrum muriaticum 30C, single dose was given. In the subsequent followups, the same was repeated once and later also prescribed in a higher potency (i.e., 200 C potency) as per the requirement of the case. Finally, at the end of one year, USG pelvis revealed no evidence of mass lesion in the uterus. As rightly said by Thomas Skinner [13], “Constitutional treatment alone was and is all that is necessary for successful treatment of all vaginal, uterine and ovarian diseases". The same holds true in this case of uterine adenomyosis too.

\section{Conclusion}

In the present case, a striking peculiarity in terms of mental general and causation, i.e., ailments from the death of her parent

\section{References}

${ }^{1}$ Ferenczy A. Pathophysiology of adenomyosis. Hum Reprod Update 1998; 4: $312-322$

2 Emge LA. The elusive adenomyosis of the uterus: its historical past and its present state of recognition. Am J Obstet Gynecol 1962; 83: 1541 - 1563

${ }^{3}$ Kilkku P, Erkkola R, Gronroos M. Nonspecificity of symptoms related to adenomyosis, a prospective comparative survey. Acta Obstet Gyn Scan 1984; 63: 229-231

${ }^{4}$ McCausland AM. Hysteroscopic myometrial biopsy: its use in diagnosing adenomyosis and its clinical application. Am J Obstet Gynecol 1992; 166: 1619-1626

${ }^{5}$ Fedele L, Bianchi S, Dorta M et al. Transvaginal ultrasonography in the diagnosis of diffuse adenomyosis. Fertil Steril 1992; 58: 94-97

${ }^{6}$ Arora A, Bhutani R, Gupta R et al. MRI findings in uterine adenomyosis - Case 8488 ; 2010, European Society of radiology teaching files, radiological case database. Online: http://www.eurorad.org/ case.php?id=8488 [accessed May 1, 2014] 7 van Zandvoort R. Complete Repertory. In Hompath Classic 8.0; 2000-2005, by Shah Jawahar, West Mumbai

${ }^{8}$ McCausland AM. Hysteroscopic myometrial biopsy: its use in diagnosing adenomyosis and its clinical implication. Am J Obstet Gynecol 1992; 166: 1619-1626

9 Stewart EA. Uterine fibroids. Lancet 2001; 357: 293-298

${ }^{10}$ Boericke W. Trillium Pendulum. New Manual of Homoeopathic Materia Medica and Repertory. New Delhi: B. Jain (P) Ltd.; 2001: 463, 628-629

${ }^{11}$ Allen HC. Genitalia Female. H.C. Allen's Key Notes and Characteristics with Comparisons with Bowel Nosodes. New Delhi: B. Jain (P) Ltd.; 2004: 295-296

12 Allen JH. The Chronic Miasm Psora and Pseudopsora. New Delhi: B. Jain (P) Ltd.; 1988: 37

${ }^{13}$ Skinner T. The Diseases of Females. New Delhi: B. Jain (P) Ltd.; 1997: 131 in the complete elimination of the adenomyosis of the uterus at the end of one year's treatment. Therefore, it can be concluded that in this case the homeopathic constitutional approach is highly effective in the cure of uterine adenomyosis.

\section{Conflict of Interest}

We declare no conflict of interest.

\author{
Dr. Y. S. Suryanarayana \\ Dr. Hima Bindu Ponnam \\ Ext. Clinical Research Unit \\ Princess Durru Shehvar Children's \& \\ General Hospital \\ Purani Haveli \\ Hyderabad 500 002, Andhra Pradesh \\ India \\ E-mail:drbindu_hima@yahoo.com
}

